\title{
Introduction to Virtual, Augmented, and Mixed Reality in Healthcare Minitrack
}

\author{
Elham Rastegari \\ Creighton University \\ elhamrastegari@creighton.edu
}

\author{
Nargess Tahmasbi \\ Pennsylvania State University \\ nvt5061@psu.edu
}

\section{Introduction}

Advancement in Virtual Reality (VR), Augmented Reality (AR), and Mixed Reality (MR) technologies has led to new avenues of delivering healthcare service to society. These technologies have variety of applications in the biomedical and medical domain: e.g., rehabilitation of patients at home, monitoring treatments in patients, and training healthcare professionals.

This minitrack covers topics that describe virtual reality integration into healthcare systems both from the design aspect and behavioral impact aspect (e.g. impact of VR adoption on patients' perception and doctors' performance).

Papers in the current minitrack focus on behavioral aspects of VR in elderly life experience (driving) and identifying depression. The research methods include qualitative approach and literature review.

The current papers expand our understanding of methodologies to apply VR technology to improve well-being of individuals and their mental health. We would like to also invite future papers that expand the theoretical aspects of application of VR in healthcare and contribute to extending related IS theories. Moreover, data-driven quantitative approaches of VR/AR/MR in healthcare are welcome in future submissions. 